\title{
A prospective study to validate the functional assessment of cancer therapy (FACT) for epidermal growth factor receptor inhibitor (EGFRI)-induced dermatologic toxicities FACT-EGFRI 18 questionnaire: SWOG S1013
}

Siu-Fun Wong ${ }^{1 *}$ (D), Joseph M. Unger ${ }^{2}$, James L. Wade III ${ }^{3}$, Lynne I. Wagner ${ }^{4}$, Mario E. Lacouture ${ }^{5}$, Keisha C. Humphries ${ }^{3}$, Anna Moseley ${ }^{2}$, Kathryn Arnold², Mario R. Velasco $\mathrm{Jr}^{3}$, Justin D. Floyd ${ }^{6,7}$, Benjamin T. Esparaz ${ }^{3}$, Afsaneh Barzi ${ }^{8}$, Heinz-Josef Lenz ${ }^{8}$, Marianna Koczywas ${ }^{9}$, Shaker Dakhil ${ }^{10}$, Gary V. Burton ${ }^{11}$, Michael J. Fisch ${ }^{12}$, N. Lynn Henry ${ }^{13}$, Dawn L. Hershman ${ }^{14}$ and Carol M. Moinpour ${ }^{15}$

\begin{abstract}
Background: Papulopustular rash is a common class effect of epidermal growth factor receptor inhibitors (EGFRI) that can affect patients' health-related quality of life and cause disruptions to treatment. SWOG S1013 (NCT01416688) is a multi-center study designed to validate the Functional Assessment of Cancer Therapy EGFRI 18 (FACT-EGFRI 18) using 7-items from the National Cancer Institute (NCl) Common Terminology Criteria for Adverse Events (CTCAE) version 4.0 to assess EGFRl-induced skin-related toxicities and their impact on functional status.

Methods: Patients with a diagnosis of colorectal or lung cancer to receive EGFRI therapies for at least 6 weeks were enrolled. Patient self-assessments using the FACT-EGFRI 18 were completed prior to undergoing CTCAE assessment by trained clinicians at baseline, weekly $\times 6$, and then monthly $\times 3$. The psychometric properties of the FACT-EGFRI 14 (skin toxicity items only) and 18 (plus 2 nail and 2 hair items) were established based on criterion validity, known groups validity, internal consistency reliability, and responsiveness to change.

Results: Of the 146 registered patients, 124 were evaluable. High Cronbach's alpha (>0.70) for both FACT-EGFRI 14 and FACT-EGFRI 18 scores across assessment times were observed. Although agreement (i.e. criterion validity) between individual and summary scales of the FACT-EGFRI 18 for assessing skin toxicity was good, agreement with the clinician-reported CTCAE was only fair. The minimal important difference was determined to be 3 points. The results also demonstrated responsiveness to symptom change.

(Continued on next page)
\end{abstract}

\footnotetext{
* Correspondence: sfwong1120@gmail.com

${ }^{1}$ Chapman University School of Pharmacy, Rinker Health Science Campus,

9401 Jeronimo Road, Irvine, CA, USA

Full list of author information is available at the end of the article
}

\section{Springer Open}

(0) The Author(s). 2020 Open Access This article is licensed under a Creative Commons Attribution 4.0 International License, which permits use, sharing, adaptation, distribution and reproduction in any medium or format, as long as you give appropriate credit to the original author(s) and the source, provide a link to the Creative Commons licence, and indicate if changes were made. The images or other third party material in this article are included in the article's Creative Commons licence, unless indicated otherwise in a credit line to the material. If material is not included in the article's Creative Commons licence and your intended use is not permitted by statutory regulation or exceeds the permitted use, you will need to obtain permission directly from the copyright holder. To view a copy of this licence, visit http://creativecommons.org/licenses/by/4.0/. 
(Continued from previous page)

Discussion: Based on the results of this multi-center validation study, the FACT-EGFRI 18 patient-reported outcome instrument provided data from the patient's perspective yielding unique information as well as complementing clinician-rated CTCAE grades, especially for the symptoms of pain, pruritus, and paronychia.

Conclusions: Good to excellent psychometric properties for the FACT-EGFRI 18 were demonstrated, supporting further use of this patient-reported outcomes measure. Additional validation with a more diverse group of patients should be conducted.

Keywords: EGFRI, FACT-EGFRI 18, Dermatologic toxicity, Papulopustular rash, Patient-reported outcome measure, Health-related quality of life, HRQL

\section{Background}

A characteristic rash has been documented to be a "class adverse effect" of agents that target the epidermal growth factor receptor (EGFR), including the monoclonal antibodies (Mab) cetuximab and panitumumab, and the tyrosine kinase small molecule inhibitor (TKI) erlotinib [1]. Approximately $90 \%$ and $75 \%$ of cancer patients receiving EGFR inhibitors (EGFRI) Mabs and TKIs, respectively, develop a papulopustular eruption within the first $2-3$ weeks after the start of therapy, and the toxicity is often dosedependent [2-4]. The eruption is characterized by inflammatory papules and pustules most often seen on the face, chest, and back but occasionally extending to the extremities; scratching due to pruritus can cause secondary infection. These lesions may resemble folliculitis or an acneiform drug eruption with tenderness and pruritus. Multiple large phase III trials of EGFRI therapies have found drug-related papulopustular rash in $75 \%$ of patients, including $8 \%$ with grades $3-4$ toxicity $[5,6]$. The discomfort and physical appearance of this common toxicity affect patients' instrumental activities of daily living (IADL) and health-related quality of life (HRQL), and can cause disruptions to treatment [7-9].

Interest in rash management has increased over time due to published data suggesting a possible relationship between the presence of rash and treatment response and/or patient survival $[8,10-13]$. The current management approach is either prophylactic with oral antibiotics and topical corticosteroids or reactive treatment with dose modifications or discontinuation of EGFRIs upon the occurrence of intolerable Grade 2 or Grade 3 or 4 skin toxicity. No standardized treatment regimen has been identified as the optimal approach to prevent or treat EGFRI-induced skin toxicity. Research to advance knowledge and establish consensus to optimally manage EGFRI-induced skin rash requires universally accepted, reliable, and validated patient reported outcome (PRO) measures to complement the National Cancer Institute Common Toxicity Criteria and Adverse Events (CTCAE) [14-16]. measures add the impact of skin toxicities and functional status assessments.
Between 2010 to 2017, three other grading systems were proposed [17-19]. None of these systems has been adopted as the standard of care tool by the medical community. The National Cancer Institute's PRO-CTCAE was an important addition to the small set of patientreported symptom measures addressing skin toxicity symptoms, but were not available when S1013 was developed [20-24]. One concern is these items do not thoroughly address the full range of skin toxicities on functional impacts. The patient-reported instrument used in this trial, the Functional Assessment of Cancer Therapy EGFRI 18 (FACT-EGFRI 18) as shown in Table 1 [25], does address these broad symptoms, but had not been fully validated. Therefore, there remained a need to validate a PRO measure to capture patients' experiences with treatment and effects on HRQL, enhance clinicians' ability to accurately assess the severity and effect of skin toxicity symptoms, and evaluate interventions to prevent or manage skin toxicities.

Because of the absence of a validated or "gold standard" PRO measure to assess EGFRI skin toxicity, a multi-center study was designed to evaluate the psychometric properties of the FACT-EGFRI 18 [25]. The goal was to obtain a more comprehensive assessment of EGFRI-induced skin-related toxicities, particularly with respect to impact on patient functional status, in order to inform and promote optimal supportive care as well as continued research for this patient population.

\section{Methods \\ Study design \\ Patients}

The trial was conducted by the SWOG (formerly the Southwest Oncology Group) Cancer Research Network, a National Cancer Institute (NCI)-sponsored National Clinical Trials Network group and a member of the NCI's Community Oncology Research Program. Eligible patients, screened and recruited by investigators from 11 SWOG sites, had a diagnosis of colorectal or lung cancer and were planning to receive one of the following EGFRI treatment regimens for at least 6 weeks: 1) Cetuximab $400 \mathrm{mg} / \mathrm{m}^{2}$ IV loading dose, $250 \mathrm{mg} / \mathrm{m}^{2}$ IV weekly; 2) 
Table 1 The Functional Assessment of Cancer Therapy-EGFRI 18 (FACT-EGFRI 18)

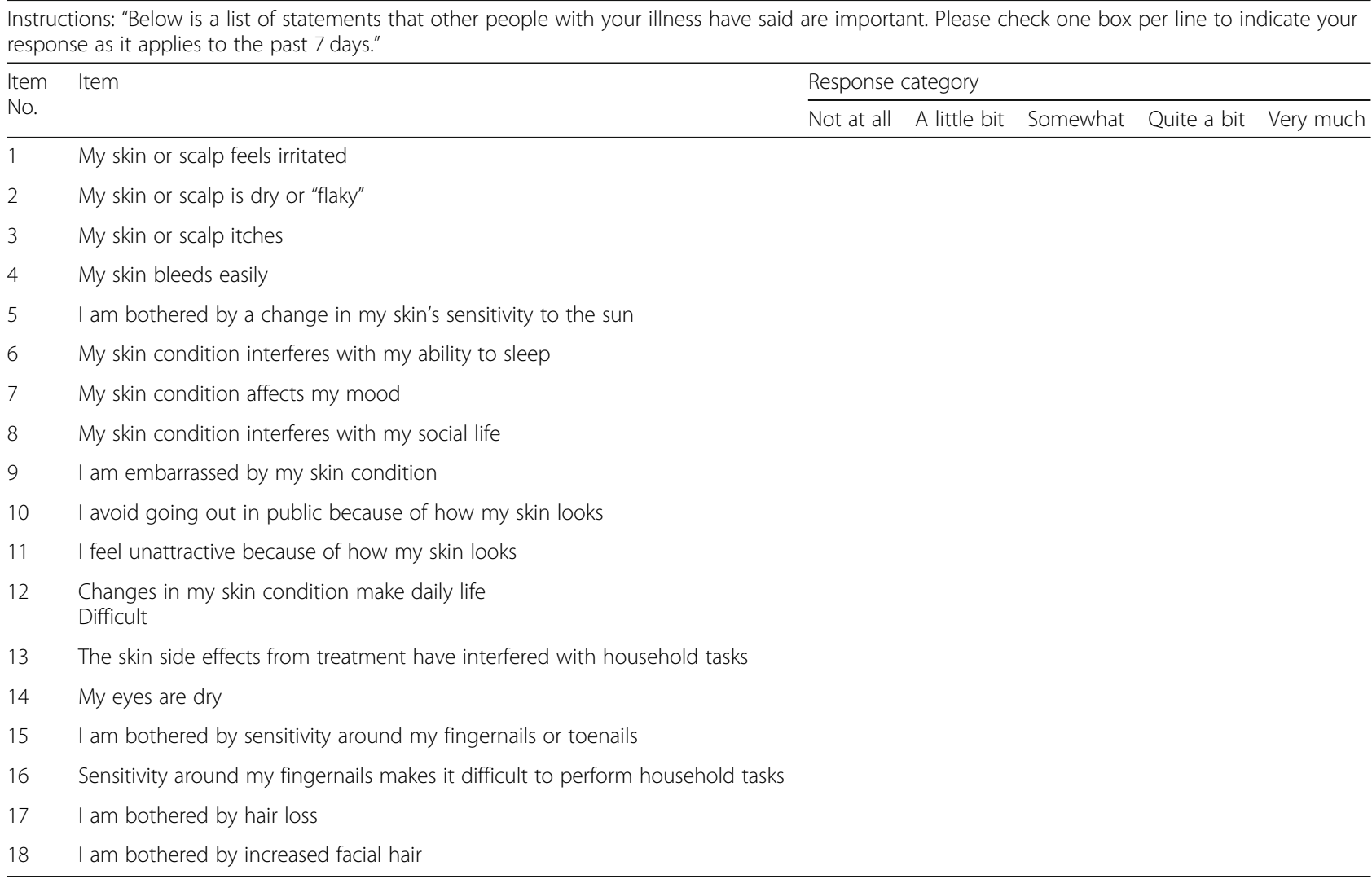

Note: FACT-EGFRI 18 measure and scoring instructions are available at the following website: https://www.facit.org/FACITOrg/Questionnaires

Cetuximab $500 \mathrm{mg} / \mathrm{m}^{2}$ IV every 2 weeks; 3) Panitumu$\mathrm{mab} 6 \mathrm{mg} / \mathrm{kg}$ IV every 2 weeks; or 4) Erlotinib 100-150 mg PO daily. Concurrent chemotherapy was allowed, except for agents known to cause rash that could interfere with EGFRI-induced skin toxicity assessment. Patients receiving prior EGFRI therapy must have fully recovered from any skin toxicities prior to registration. Patients must not have been planning to receive concomitant medications or treatments that could cause rash or other dermatologic reactions (including radiation therapy) and must not have any serious concomitant skin disorder (such as eczema) that could interfere with assessment of EGFRI-induced skin toxicity. Patients had a Zubrod [26] performance status of $0-2$. Patients completed questionnaires in English.

All patients were informed of the investigational nature of this study and were required to sign and give written informed consent in accordance with institutional and federal guidelines.

Patient-completed measures: FACT-EGFRI 18 questionnaire The FACT-EGFRI 18 [25] is a PRO measure to assess dermatologic-related symptom burden and HRQL among patients receiving EGFRIs. It includes 18 items evaluating the physical, functional, emotional, and social impact of symptoms in three anatomical areas: skin with 14 items, which comprises the FACT-EGFRI 14, plus 2 nail items and 2 hair items. In accordance with standard FACT administration procedures [27], patients rate each item using a 5-point Likert scale from 0 (Not at all) to 4 (Very much) based on the past 7 days. Based on the Functional Assessment of Chronic Illness Therapy (FACIT) scoring system, a higher score indicates better HRQL (e.g. lower severity of symptoms) [25]. Patient assessments were completed prior to physician assessments at each visit. The assessment timepoints occurred prior to EGFRI initiation at baseline (Day 1) and on Days 8, 15, 22, 29, 36, and 43; and then monthly for 3 months on Days 71, 99 and 127. Assessments continued through Day 127 even if EGFRI treatment was delayed or discontinued.

\section{Patient-completed measures: anchor items}

This two-item patient completed measure addresess the patient's sense of the degree of change in the severity of skin symptoms and the degree of change in the impact of skin symptoms on the patient's daily life since the last time questionnaires were completed [28]. These two items were used to help estimate a mean level of change 
in symptom severity and impact experienced by patients in S1013. The severity item response for minimal level of change was a little bit better or a little bit worse (representing a one-point change in either direction). Selection of zero reflected the patient's sense that symptom severity was about the same. Very much change (very much better or worse) was represented by selection of + 2 or -2 . Instead of the mean minimum difference, the overall mean difference is reported. The same definitions held for the change in the impact of skin symptoms on daily life. Patients did not complete the anchor items at the prestudy assessment time but began completing this form with the second assessment at day 8 . The mean score for each anchor choice through day 43 for severity and impact was calculated for a single mean score for change in severity and change in impact. The results reported in this study intend to emphasize the mean overall change in skin toxicity symptom severity and skin toxicity symptom impact and not the mean minimum difference.

\section{Clinician-completed measures: CTCAE items}

Seven National Cancer Institute Common Toxicity Criteria and Adverse Events (CTCAE) items [15] (Rash acneiform, Pruritus, Dry skin, Pain of skin, Paronychia, Alopecia, and Hypertrichosis) addressed criterion validity for the FACT-EGFRI-18 (Online Appendix Table 1). These seven items are most commonly associated with skin toxicity assessment. The grading of the CTCAE ranges from Grades 1 through 5 with unique clinical descriptions of severity for each adverse event defined as: Grade $1=$ Mild, Grade $2=$ Moderate, Grade $3=$ severe, Grade $4=$ Life-threatening or disabling, and Grade $5=$ Death. Although the NCI CTCAE items do not have psychometric documentation similar to that provided for patient-reported measures, they are widely accepted as physician/clinician measures of adverse events in cancer clinical trials. To minimize variability in the ratings, only physician investigators and the nurse/clinical research associate at each site who had previously undergone formal training in the CTCAE assessments by SWOG rated the subjects' EGFRI-induced skin toxicities.

\section{Clinician-completed measure: performance status items}

The Zubrod Scale is the performance status (PS) measure developed by CG Zubrod for use in cancer clinical trials [26]. It is the measure used in the Eastern Cooperative Oncology Group (ECOG) - American College of Radiology Imaging Network (ACRIN) trials [29]. This PS measure is a 5-point scale from normal status to unable to get out of bed. In S1013, the Zubrod provided a "history" of PS over the course of the study.

\section{Statistical considerations}

The overall goal of this study was to evaluate the psychometric properties of the FACT-EGFRI 18 [25] based on criterion validity, known groups validity, internal consistency reliability, and responsiveness to change. To be considered evaluable for endpoint analyses, patients must have met all eligibility criteria, completed the baseline FACT-EGFRI 18 prior to the start of EGFRI treatment, and had valid anchor assessments (Days 1, 8, 15, or 22) and valid follow-up assessments (Days 29, 36, or 43). A valid assessment is one where the FACT-EGFRI 18 is completed prior to the physician's evaluation of skin toxicity. The analysis focused on the psychometric properties of the FACT-EGFRI 18 [25] through the Day 43 assessment, corresponding to the minimum required treatment time for eligibility.

\section{Criterion validity}

Because there is no validated or "gold standard" PRO measure available in this setting, criterion validity was defined as agreement between the FACT-EGFRI 18 [25] and the CTCAE [15] scoring system. Agreement was measured using an unweighted Kappa statistic, with moderate or better agreement defined as Kappa [30] coefficients of $\geq 0.41$. Agreement was assessed between the seven treatment toxicity categories most commonly associated with EGFRI toxicity and the total and individual scores from the FACT-EGFRI 18 items. To enable comparison between the EGFRI score and the CTCAE grade using a Kappa statistic [30], total scores for the EGFRI categories were generated on a scale from 0 to 100 . These scores were then categorized into the number of respective CTCAE levels using ranks, with cell sizes defined by the patients' corresponding CTCAE score distributions. Constructing marginal distributions for EGFRI total scores in this fashion avoids arbitrary categorization (since it relies on the observed distribution of CTCAE scores) and also allows for the contingency of perfect concordance (a desirable property, which is highly unlikely). Due to the fact that patients could enter this study with none to any level of skin toxicity and that approximately $80 \%$ of patients receiving the EGFRI agents develop skin toxicity, we expected to observe a broad range of such toxicities including none. Only the sixteen items that explicitly corresponded to CTCAE items were used for the criterion validity analyses. A table showing items from both measures is included in the online appendix.

\section{Known groups validity}

Known groups validity [31-33] was assessed by examining differences in mean EGFRI subscores (eg. rash, pruritus, pain) between patients who reported a PS of 0 (indicating normal activity without symptoms) and patients who reported feeling any symptoms $(\mathrm{PS}>0)$. 
ANOVA was used to compare FACT-EGFRI 18 scores between groups, with differences of $1 / 3$ to $1 / 2$ of a standard deviation considered of interest [31]. The a priori hypothesis was that mean scores of the FACTEGFRI 18 skin adverse events measure would be significantly higher (better) for lower (better) performance status (based on reported symptom level).

\section{Internal consistency reliability}

Internal consistency reliability was evaluated using Cronbach's alpha [34]. Coefficients of $>0.70$ are considered sufficient evidence of reliability [35]. Reliability was assessed for two scores: the FACT-EGFRI 14 score for the 14 skin toxicities and the full FACT-EGFRI 18 scale that included two nail and two hair toxicity items in addition to the 14 skin toxicities.

\section{Responsiveness to change}

Longitudinal regression using linear mixed models (wth patient treated as a random effect) was used to measure change in FACT-EGFRI 18 scores over time, with $1 / 3$ to $1 / 2$ of a standard deviation reflecting a clinically significant change [31].

\section{Minimal important difference (MID)}

The MID for the FACT-EGFRI 18 scores was estimated by comparing unweighted mean changes in the FACTEGFRI 18 score to the patient's direct assessment of change between adjacent levels of two anchor items, severity and impact [28].

Criterion validity, known groups validity and reliability were described at each timepoint and overall. Secondary objectives included the assessment of minimally important change and the association of the FACT-EGFRI 18 scores with treatment profiles.

\section{Power and sample size}

The primary objective was to establish the validity of the new measure through the collection of psychometric properties. The accrual goal was $N=140$ eligible patients, among whom $80 \% \quad(N=112)$ were expected to be evaluable for psychometric endpoints. For a Kappa [36] of 0.7 and 5 levels and a sample size of 112 evaluable patients, there is $95 \%$ confidence that the true agreement was at least 0.60 ("good" agreement), assuming equal levels of agreement and disagreement for each of the category levels. Given similar parameters, for a Kappa of 0.4, there is 95\% confidence that the true agreement was at least 0.28 ("fair" agreement).

\section{Results}

Research staff at 11 institutions enrolled 146 patients between November 15, 2011 and October 1, 2016. Two patients were ineligible: one patient had baseline performance status greater than 2 and the other did not have colorectal or lung cancer. Twenty additional patients were not evaluable (Fig. 1), leaving 124 patients evaluable for the analysis of psychometric endpoints. Evaluable patients were $47 \%(n=58)$ male, 16\% $(n=20)$ non-white and $10 \%(n=12)$ Hispanic, with a median age of 65 years (range 32-88 years). Thirty one patients (25\%) had performance status of 0 and $75 \%(n=93)$ had performance status $>0$. Fifty-four percent $(n=67)$ of evaluable patients were treated with erlotinib, $15 \%(n=$ 18 ) with each of the 2 different dose regimens of cetuximab, and $17 \%(n=21)$ with panitumumab. Papulopustular rash developed in 112 (90\%) of the evaluable patients. Maximum per-patient rash severity ratings based on CTCAE grading were predominantly reported at grade 1 $(47 \%, n=58)$ and grade $2(43 \%, n=53)$, while $10 \%$ $(n=12)$ reported grade 3; the median onset of the rash occurred at 2 weeks. Descriptive statistics for the item and scale scores of the FACT-EGFRI 18 are shown in Table 2.

\section{Criterion validity}

Kappa statistics ranged from -0.02 to 0.53 , varying by assessment time and skin symptom (Fig. 2). Overall, 26 of the 46 (57\%) individual assessment-specific Kappa statistics were $>0.20$, indicating fair agreement between

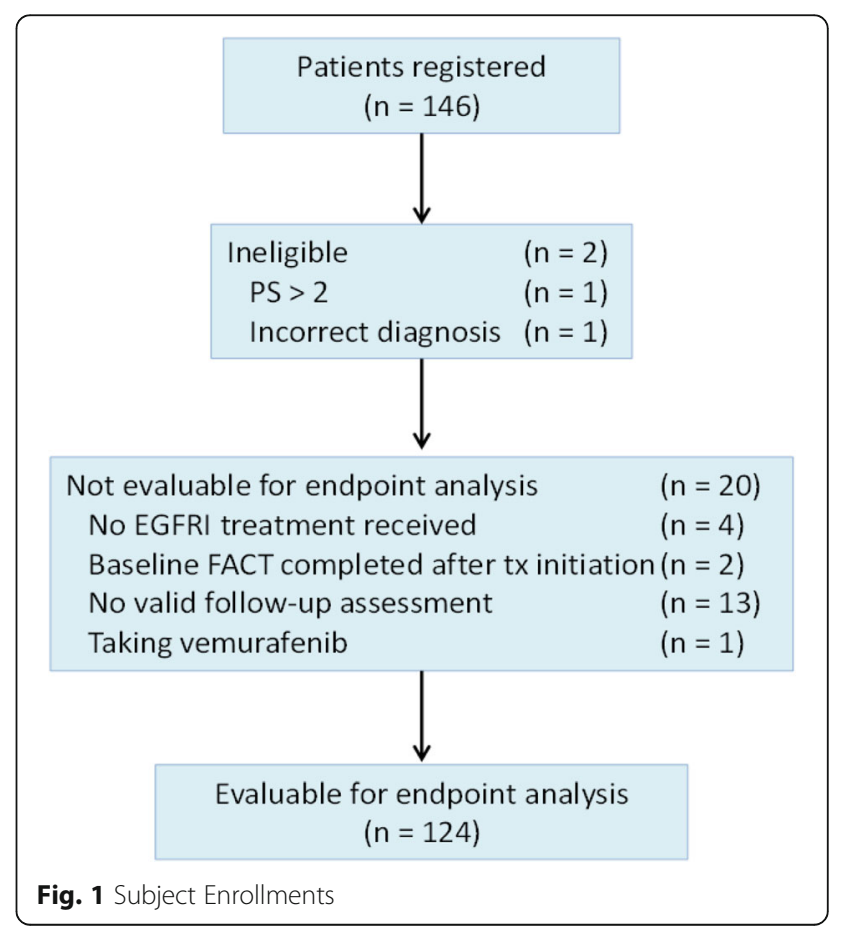


Table 2 Descriptive Statistics for Total FACT-EGFRI 18 Scale and Subscale Scores*

\begin{tabular}{|c|c|c|c|c|c|c|c|c|c|c|}
\hline & \multicolumn{10}{|l|}{$\begin{array}{l}\text { Mean } \\
\text { (SD) }\end{array}$} \\
\hline & Day 1 & Day 8 & Day 15 & Day 22 & Day 29 & Day 36 & Day 43 & Day 71 & Day 99 & Day 127 \\
\hline \multirow[t]{2}{*}{ Alopecia } & 92.4 & 93.7 & 93.5 & 93.6 & 91.5 & 90.1 & 88.3 & 82.8 & 79.9 & 75.0 \\
\hline & $(18.4)$ & $(16.5)$ & $(16.5)$ & $(17.5)$ & $(17.9)$ & (18.0) & $(21.7)$ & $(26.6)$ & $(28.5)$ & (30.6) \\
\hline \multirow[t]{2}{*}{ Dry skin } & 94.4 & 90.1 & 82.1 & 83.6 & 86.3 & 88.3 & 86.6 & 85.0 & 84.5 & 85.6 \\
\hline & (8.7) & (13.9) & $(21.7)$ & $(19.3)$ & (16.9) & $(11.5)$ & $(12.6)$ & $(16.1)$ & $(13.4)$ & (17.4) \\
\hline \multirow[t]{2}{*}{ Hypertrichosis } & 96.7 & 96.1 & 94.3 & 95.4 & 97.4 & 97.3 & 95.8 & 87.8 & 91.7 & 86.4 \\
\hline & (11.1) & (13.8) & $(17.4)$ & $(11.9)$ & (8.6) & (8.9) & $(12.4)$ & $(23.6)$ & $(22.4)$ & $(24.3)$ \\
\hline \multirow[t]{2}{*}{ Pain of skin } & 95.5 & 86.6 & 76.1 & 79.9 & 82.4 & 86.9 & 82.7 & 83.0 & 85.4 & 84.3 \\
\hline & (11.4) & $(18.4)$ & $(23.7)$ & $(22.7)$ & $(20.0)$ & $(12.2)$ & $(16.9)$ & $(18.5)$ & $(16.6)$ & $(18.5)$ \\
\hline \multirow[t]{2}{*}{ Paronychia } & 96.2 & 95.2 & 92.5 & 87.7 & 86.8 & 88.0 & 82.4 & 77.2 & 80.2 & 75.8 \\
\hline & (9.9) & $(10.3)$ & $(14.2)$ & $(20.6)$ & $(20.3)$ & $(16.4)$ & $(22.6)$ & $(25.3)$ & $(26.3)$ & $(24.6)$ \\
\hline \multirow[t]{2}{*}{ Pruritus } & 92.8 & 83.0 & 74 & 76.5 & 78.8 & 84.9 & 79.1 & 80.6 & 79.2 & 81.4 \\
\hline & $(15.1)$ & $(22.4)$ & $(24.2)$ & $(23.4)$ & $(21.4)$ & (12.8) & $(18.3)$ & $(18.6)$ & $(19.4)$ & $(20.5)$ \\
\hline \multirow[t]{2}{*}{ Rash } & 94.9 & 89.5 & 80.7 & 82.8 & 85.5 & 88.5 & 85.2 & 84.4 & 84.3 & 84.7 \\
\hline & $(8.2)$ & (13.9) & $(20.7)$ & (18.9) & (15.8) & $(9.7)$ & $(13.1)$ & $(16.6)$ & $(14.5)$ & $(16.9)$ \\
\hline \multirow[t]{2}{*}{ Total FACT- EGFRI 18} & 67.9 & 64.9 & 60.1 & 60.8 & 62.1 & 63.8 & 61.5 & 60.1 & 60.0 & 59.6 \\
\hline & (5.3) & (8.1) & (11.8) & (10.8) & (9.0) & (6.0) & (8.6) & $(11.0)$ & (9.9) & (11.6) \\
\hline
\end{tabular}

*Higher FACT-EGFRI scores reflect better health-related quality of life

FACT-EGFRI 18 scores and CTCAE scores. Moderate agreement $(\geq 0.41)$ for selected timepoints was found for pain of skin, paronychia, and pruritus. Mean toxicityspecific Kappa scores exceeded 0.2 for pain of skin, paronychia, and pruritus only.

\section{Known groups validity}

Results of the ANOVA analysis are summarized in Fig. 3. Since higher FACT-EGFRI 18 subscores indicate better HRQL, positive standardized values (effect sizes) 0.33 or higher (MID) were considered of interest. Overall, 32 of the $56(57 \%)$ individual assessment-specific results were $\geq 0.33$. Mean subscale scores were at least 0.33 for dry skin, pain of skin, pruritus, and rash acneiform, as well as for the total FACT-EGFRI 18 score. The full FACT-EGFRI 18 item scale was used to assess known groups validity, responsiveness to expected change, and reliability.

\section{Internal consistency reliability}

Cronbach's alpha statistics are summarized in Fig. 4 by score and by time point. Reliability estimates were uniformly high $(>0.70)$ for both the FACT-EGFRI 14 and FACT-EGFRI 18, indicating strong internal consistency reliability, with every assessment time showing a Cronbach's alpha $>0.70$ for each scale.

\section{Responsiveness to change}

Figure 5 shows the linear mixed model fitted regression line results for responsiveness to change. The FACTEGFRI 18 total score decreased significantly over time, consistent with a decrease in HRQL over time. Subscale scores decreased significantly $(p<0.001)$ over time for dry skin, rash acneiform, paronychia, pain of skin, and pruritus, but not for hypertrichosis $(p=0.42)$ or alopecia $(p=0.12)$.

\section{Minimal important difference (MID)}

The mean difference between levels was 3.18 points for change in severity of skin symptoms, and 3.37 points for change in impact of skin symptoms (Table 3). Therefore, a reasonable estimate of the MID in the FACT-EGFRI 18 scores is 3 points. This estimate can be used to indicate the MID in future studies that include the FACTEGFRI 18 measure [28].

\section{Association with treatment profiles}

Patients who experienced a disruption in EGFRI treatment had lower FACT-EGFRI 18 scores than patients who did not have a disruption in EGFRI treatment by the end of the baseline period at assessment Day 22 (57.8 vs.62.2) and the end of the follow-up period at Day 43 (57.2 vs. 63.9). 


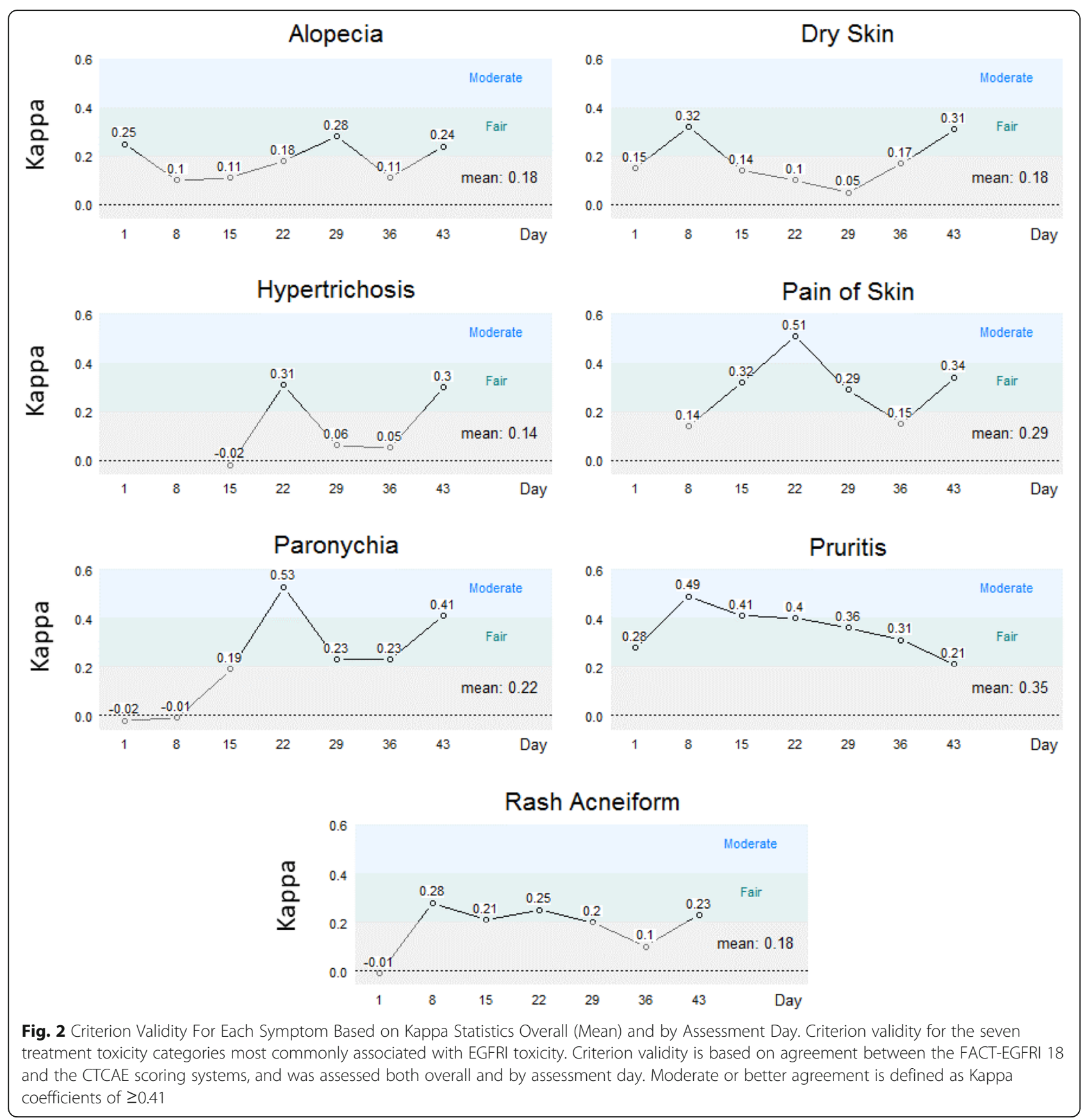

\section{Discussion}

When evaluating treatment regimens such as EGFRI that cause considerable skin toxicity that can negatively impact HRQL, it is important to have a validated instrument to assess the patient perspective. Our study showed uniformly high Cronbach's alpha $(>0.70)$ for both FACT-EGFRI 14 (skin symptoms only) and FACT-EGFRI 18 across assessment times, indicating strong evidence of internal consistency among the items that make up these two composite scores. The results also demonstrated responsiveness to symptom change, and were correlated with treatment profiles. The known groups validity results were of interest for dry skin, pain of skin, pruritus, rash, and the total FACT-EGFRI 18 score. Although agreement (i.e. criterion validity) between individual and summary scales of the FACT-EGFRI 18 and the current clinician measure for assessing EGFRI skin toxicity (physician-rated CTCAE items) was generally only fair, this may reflect the limitations of the 


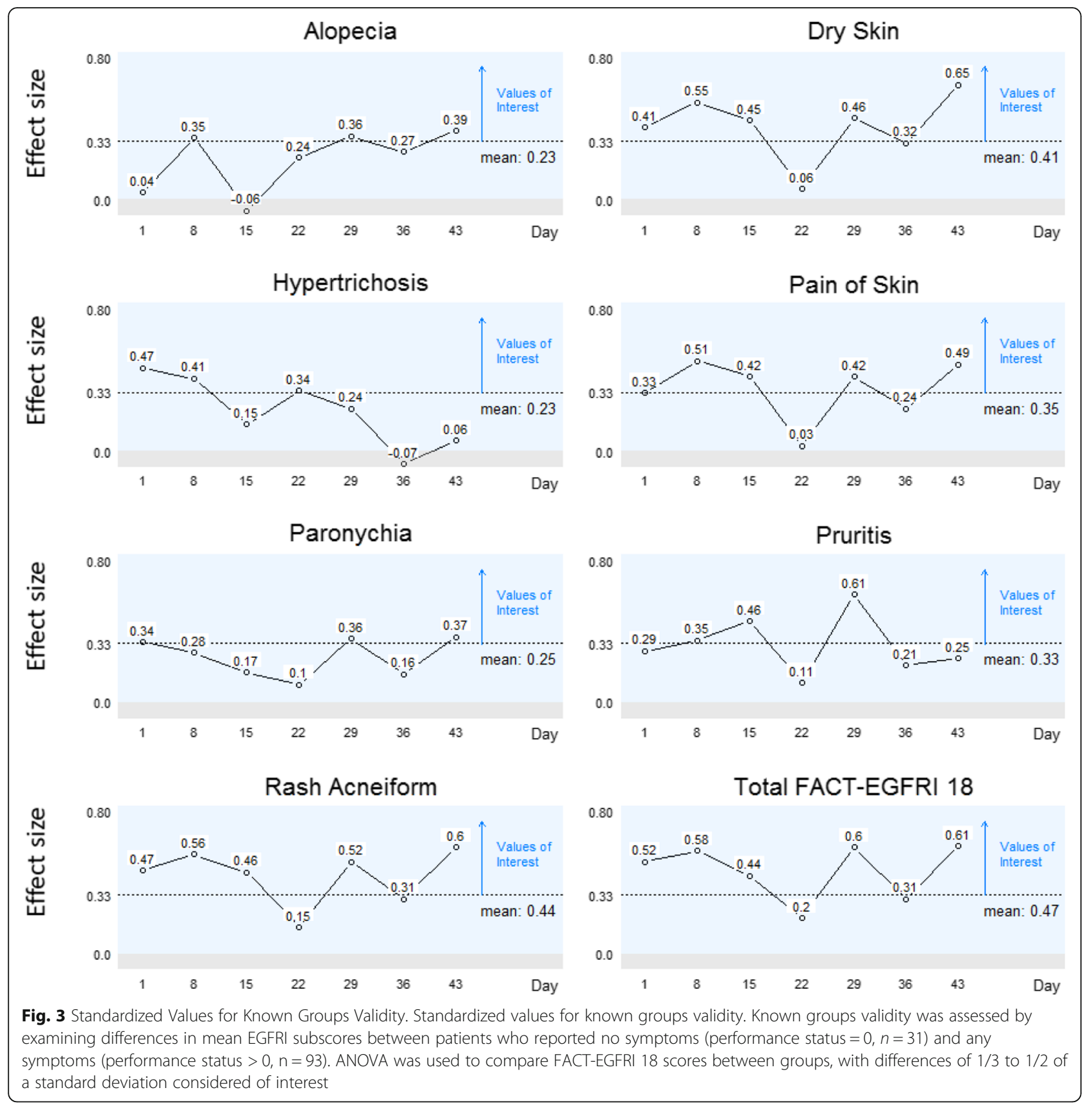

physician-reported CTCAE measure itself. The MID for the FACT-EGFR 18 was determined to be 3 points. The 2 items that reached the moderate agreement $(\geq 0.41)$ for the criterion validity analysis were pain and pruritus, which are non-measurable and non-observable symptoms. Therefore, the inclusion of these 2 important skin-related symptoms in a PRO measure will be informative.

As previously noted, there are no currently available standardized PRO measures to quantify EGFRIinduced dermatologic adverse events and impact on functional status, from the patient's perspective. Furthermore, the subjective nature of some of these EGFRI-induced papulopustular rash symptoms (i.e. pain of skin and itching) make it more appropriate and accurate to be assessed using the PRO approach. Dermatology-specific PROs such as the Skindex-29 [37] and the Dermatology Life Quality Index [38] are available and widely used, but these tools are not specifically designed to assess EGFRI-associated toxicity, such as hair change and paronychia. Therefore our results provide important data to support using the 


\section{Cronbach's Alpha}

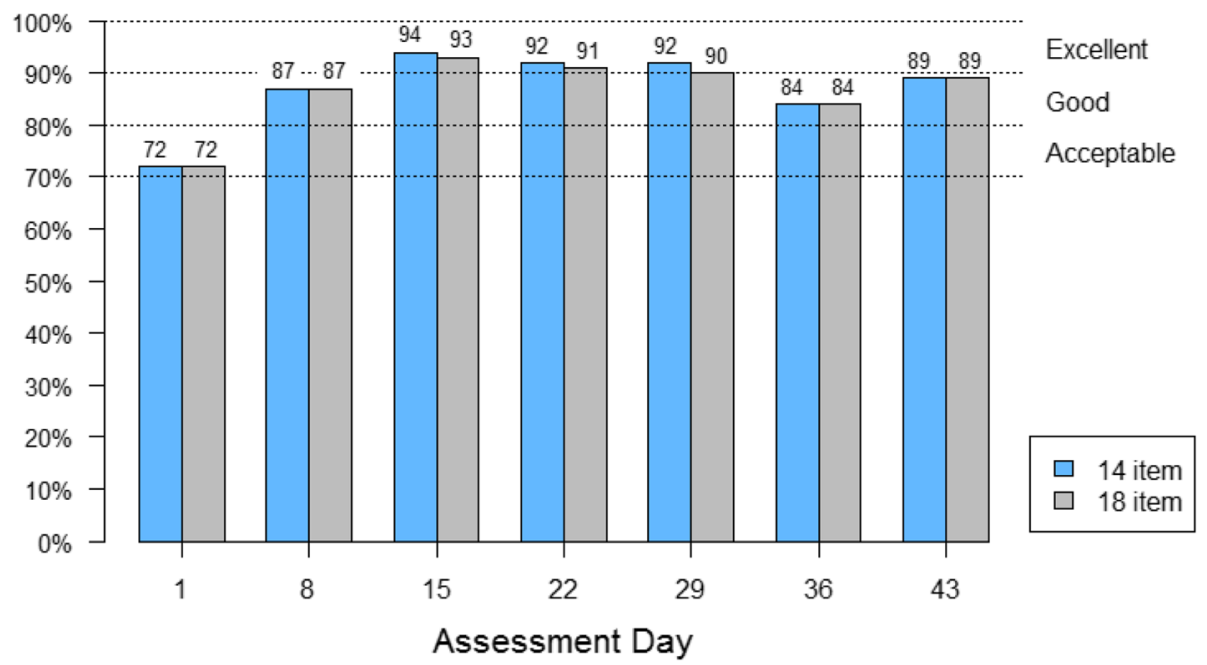

Fig. 4 Cronbach's alpha. Cronbach's alpha is a measure of internal consistency reliability. Reliability was assessed for the FACT-EGFRI 14 14-item score for the 14 skin toxicities and the full FACT-EGFRI 18 scale of 18 items. Coefficients of $>0.70$ are considered sufficient evidence of reliability

FACT-EGFRI 18 in EGFRI-treated patients to assess the impact of this specific class of treatment on HRQL.

This validation study included patients from across multiple oncology practice sites throughout the United States treated with a number of different EGFRI- containing treatment regimens. The included population was consistent with published experience with these agents, including incidence and time to development of the papulopustular rash [2-4]. However it included fewer than 20\% non-white patients and was limited to English speaking patients, which somewhat limits generalizability.

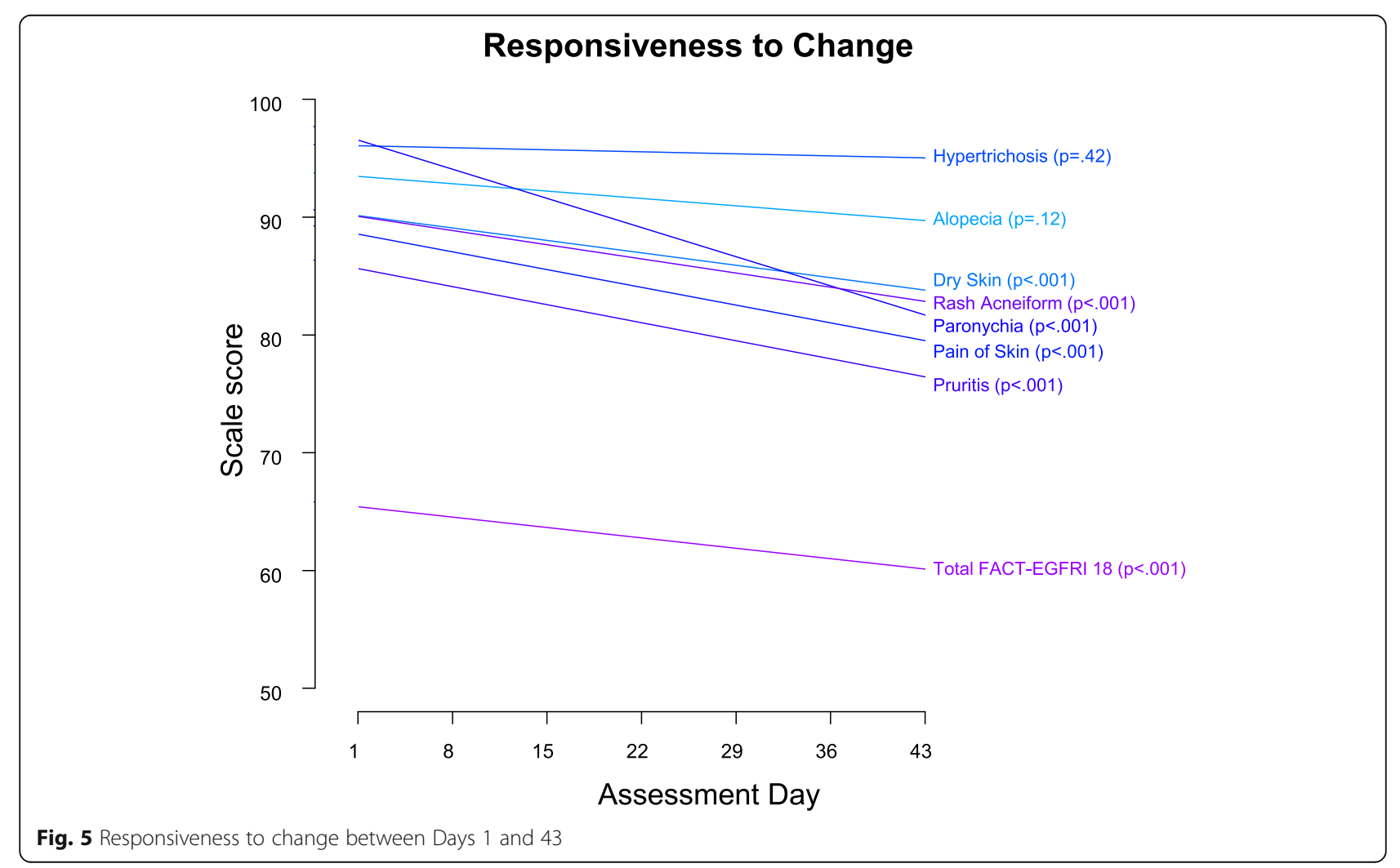


Table 3 Mean Change in FACT-EGFRI by Patient-Reported Change in Severity and Impact of Skin Symptoms

\begin{tabular}{|c|c|c|c|c|c|c|c|c|c|c|c|}
\hline \multirow{3}{*}{$\begin{array}{l}\text { Assessment } \\
\text { time }\end{array}$} & \multicolumn{11}{|c|}{ FACT EGFRI Total Score } \\
\hline & \multicolumn{2}{|c|}{ A lot better } & \multicolumn{2}{|c|}{ A little bit better } & \multicolumn{2}{|c|}{ About the same } & \multicolumn{2}{|c|}{ A little bit worse } & \multicolumn{2}{|c|}{ A lot worse } & \multirow[t]{2}{*}{ Mean Diff-erence ${ }^{a}$} \\
\hline & Mean & $\mathrm{N}$ & Mean & $\mathrm{N}$ & Mean & $\mathrm{N}$ & Mean & $\mathrm{N}$ & Mean & $\mathrm{N}$ & \\
\hline \multicolumn{12}{|c|}{ Change in SEVERITY of skin symptoms } \\
\hline Day 8 & & & -0.50 & 4 & 0.86 & 69 & -6.36 & 33 & -17.53 & 11 & \\
\hline Day 15 & 4.50 & 2 & -1.71 & 12 & 0.15 & 45 & -6.96 & 47 & -14.56 & 16 & \\
\hline Day 22 & 4.43 & 14 & 3.98 & 18 & -0.18 & 50 & -3.67 & 36 & -1.33 & 3 & \\
\hline Day 29 & 5.76 & 20 & 3.00 & 23 & 0.21 & 55 & -2.03 & 21 & -2.5 & 2 & \\
\hline Day 36 & 4.01 & 16 & 2.49 & 28 & -0.31 & 48 & 0.53 & 22 & -8 & 1 & \\
\hline Day 43 & 2.20 & 17 & 0.64 & 19 & 0.04 & 54 & -4.52 & 21 & -7.25 & 4 & \\
\hline Mean & 4.17 & & 1.32 & & 0.13 & & -3.84 & & -8.53 & & 3.18 \\
\hline \multicolumn{12}{|c|}{ Change in IMPACT of skin symptoms on daily life } \\
\hline Day 8 & & & -1.33 & 3 & 0.05 & 84 & -9.17 & 23 & -20.5 & 6 & \\
\hline Day 15 & 4.5 & 2 & -0.4 & 10 & -1.8 & 66 & -8.73 & 36 & -17.13 & 8 & \\
\hline Day 22 & 3.36 & 14 & 8.44 & 9 & -0.75 & 79 & -4.91 & 17 & 4 & 2 & \\
\hline Day 29 & 7.43 & 14 & 2.86 & 21 & 0.44 & 70 & -2.57 & 14 & -5 & 2 & \\
\hline Day 36 & 4.22 & 16 & 2.19 & 18 & 0.42 & 70 & -1.2 & 11 & & & \\
\hline Day 43 & 1.91 & 18 & 1.23 & 13 & -0.7 & 71 & -5.1 & 10 & -7.33 & 3 & \\
\hline mean & 4.28 & & 2.17 & & -0.39 & & -5.28 & & -9.19 & & 3.37 \\
\hline
\end{tabular}

anweighted mean difference calculated as the average of the difference between adjacent levels of patient-reported change categories. For instance, for severity, the difference is calculated as: mean of $[4.17-1.32,1.32-0.13,0.13-(-3.84),-3.84-(-8.53)]$

Overall these data are applicable to most community care settings and will be important for future clincial trials that include assessment with the FACT-EGFRI 18.

The FACT-EGFRI 18 provides data from the patient's perspective yielding unique information as well as complementing clinician-rated CTCAE grades, especially for the symptoms of pain, pruritus, and paronychia. With the introduction of the NCI PRO-CTCAE since the initiation of this trial, additional research should be considered to optimize the utility of NCI PRO-CTCAE items in combination with the FACT-EGFRI 18 to best evaluate the EGFRI-induced papulopustular rash.

In conclusion, the psychometric results for the FACTEGFRI 18 obtained in S1013 are supportive of use of this measure in English-speaking patients with cancer undergoing treatment with EGFRI-containing treatment regimens.

\section{Supplementary information}

Supplementary information accompanies this paper at https://doi.org/10. 1186/s41687-020-00220-X.

Additional file 1.

\section{Abbreviations}

ACRIN: American College of Radiology Imaging Network; CTCAE: Common Toxicity Criteria and Adverse Events; ECOG: Eastern Cooperative Oncology Group; EGFR: Epidermal Growth Factor Receptor; EGFRI: Epidermal Growth Factor Receptor Inhibitor; FACT: Functional Assessment of Cancer Therapy;
FACIT: Functional Assessment of Chronic Illness Therapy; HRQL: Healthrelated Quality of Life; IADL: Instrumental Activities of Daily Living; MID: Minimal Important Difference; PRO: Patient Reported Outcome; PS: Performance Status; TKI: Tyrosine Kinase Small Molecule Inhibitor

\section{Acknowledgements}

The authors wish to acknowledge and thank the patients who shared their experiences with our research team. Appreciation also goes out to the investigator team at each of SWOG study sites that facilitated subject recruitment and contributed to the study data.

\section{Authors' contributions}

SFW, JU, JW, MF, NLH, and CM contributed to the study design; data interpretation; manuscript development and manuscript review. $\mathrm{KH}, \mathrm{JF}, \mathrm{BE}$, $A B, H J L, M K, S D$, and GB contributed to the data collection and manuscript review. AM and KA contributed to the data analysis and interpretation; manuscript development and manuscript review. LW, ML, and DH contributed to the manuscript review. The authors read and approved the final manuscript.

\section{Funding}

Research reported in this publication was supported by the National Cancer Institute of the National Institutes of Health under the following grant award number: UG1CA189974 (C.D. Blanke, MD, PI). The content is solely the responsibility of the authors and does not necessarily represent the official views of the National Institutes of Health.

\section{Availability of data and materials}

The datasets generated and analyzed during the current study are not publicly available to help maintain confidentiality but are available from the SWOG data repository located at the SWOG Statistical Center in Seattle, WI.

Ethics approval and consent to participate

This study was reviewed and approved by the Institutional Review Board at each of the SWOG study sites. 


\section{Consent for publication}

All subjects participated in this study signed the Informed Consent approved by the respective Institutional Review Board to have the results of this study published in medical journals. The consent form informed patients that no personal information will be revealed.

\section{Competing interests}

The authors declare that they have no competing interests.

\section{Author details}

'Chapman University School of Pharmacy, Rinker Health Science Campus, 9401 Jeronimo Road, Irvine, CA, USA. ${ }^{2}$ SWOG Statistics and Data Management Center/Fred Hutchinson Cancer Research Center, Seattle, WA, USA. ${ }^{3}$ Heartland Cancer Research NCORP/Cancer Care Specialists of Central Illinois, Decatur, IL, USA. ${ }^{4}$ Wake Forest School of Medicine, Winston Salem, NC, USA. ${ }^{5}$ Memorial Sloan-Kettering Cancer Center, New York, NY, USA. ${ }^{6}$ Wichita NCORP, Wichita, KS, USA. ${ }^{7}$ Heartland Cancer Research NCORP/ Cancer Care Specialists of Central Illinois, Swansea, IL, USA. ${ }^{8}$ University of Southern California, Los Angeles, CA, USA. ${ }^{9}$ City of Hope, Duarte, CA, USA. ${ }^{10}$ Wichita NCORP/ Cancer Center of Kansas, Wichita, Wichita, KS, USA. ${ }^{11}$ Gulf South MU NCORP/Louisiana State University HSC-Shreveport, Shreveport, LA, USA. ${ }^{12}$ Aim Specialty Health, Chicago, IL, USA. ${ }^{13}$ University of Michigan Medical School, Ann Arbor, MI, USA. ${ }^{14}$ Columbia University Medical Center, New York, NY, USA. ${ }^{15}$ Fred Hutchinson Cancer Research Center, Public Health Sciences Division (Emerita), Seattle, WA, USA.

\section{Received: 24 February 2020 Accepted: 18 June 2020}

\section{Published online: 08 July 2020}

\section{References}

1. Busam, K. J., Capodieci, P., Motzer, R., Kiehn, T., Phelan, D., \& Halpern, A. C. (2001). Cutaneous side-effects in cancer patients treated with the antiepidermal growth factor receptor antibody C225. The British Journal of Dermatology, 144(6), 1169-1176.

2. Soulieres, D., Senzer, N. N., Vokes, E. E., Hidalgo, M., Agarwala, S. S., \& Siu, L. L. (2004). Multicenter phase II study of erlotinib, an oral epidermal growth factor receptor tyrosine kinase inhibitor, in patients with recurrent or metastatic squamous cell cancer of the head and neck. Journal of Clinical Oncology, 22(1), 77-85.

3. Baselga, J., Pfister, D., Cooper, M. R., et al. (2000). Phase I studies of antiepidermal growth factor receptor chimeric antibody C225 alone and in combination with cisplatin. Journal of Clinical Oncology, 18(4), 904-914.

4. Tarceva Package Insert. (2008). OSI pharmaceuticals, Inc and Genentech, Inc.

5. Sobrero, A. F., Maurel, J., Fehrenbacher, L., et al. (2008). EPIC: Phase III trial of cetuximab plus irinotecan after fluoropyrimidine and oxaliplatin failure in patients with metastatic colorectal cancer. Journal of Clinical Oncology, 26(14), 2311-2319.

6. Wheatley-Price, P., Ding, K., Seymour, L., Clark, G. M., \& Shepherd, F. A. (2008). Erlotinib for advanced non-small-cell lung cancer in the elderly: An analysis of the National Cancer Institute of Canada clinical trials group study BR.21. Journal of Clinical Oncology, 26(14), 2350-2357.

7. Molinari, E., De Quatrebarbes, J., Andre, T., \& Aractingi, S. (2005). Cetuximabinduced acne. Dermatology., 211(4), 330-333.

8. Perez-Soler, R., \& Saltz, L. (2005). Cutaneous adverse effects with HER1/EGFRtargeted agents: Is there a silver lining? Journal of Clinical Oncology, 23(22), 5235-5246.

9. Wagner, L. I., \& Lacouture, M. E. (2007). Dermatologic toxicities associated with EGFR inhibitors: The clinical psychologist's perspective. Impact on health-related quality of life and implications for clinical management of psychological sequelae. Oncology (Williston Park), 21(11 Suppl 5), 34-36.

10. Perez-Soler, R. (2006). Rash as a surrogate marker for efficacy of epidermal growth factor receptor inhibitors in lung cancer. Clinical Lung Cancer, 8(Suppl 1), S7-S14

11. Perez-Soler, R., \& Van Cutsem, E. (2007). Clinical research of EGFR inhibitors and related dermatologic toxicities. Oncology (Williston Park), 21(11 Suppl 5), 10-16.

12. Racca, P., Fanchini, L., Caliendo, V., et al. (2008). Efficacy and skin toxicity management with cetuximab in metastatic colorectal cancer: Outcomes from an oncologic/dermatologic cooperation. Clinical Colorectal Cancer, 7(1), 48-54.
13. Saltz, L., Kies, M., Abbruzzese, J. L., Azarnia, N., \& Needle, M. (2003). The presence and intensity of the cetuximab-induced acne-like rash predicts increased survival in studies across multiple malignanciesPaper presented at: 2003 ASCO Annual Meeting.

14. National Cancer Institute NIH, US Department of Health Human Services. (2006). Common terminology criteria for adverse events (CTCAE) version 3.0 http://ctep.cancer.gov/protocolDevelopment/electronic_applications/docs/ ctcaev3.pdf .

15. National Cancer Institute NIH, US Department of Health and Human Services. (2009). Common terminology criteria for adverse events, version 4.0 https://evs.nci.nih.gov/ftp1/CTCAE/CTCAE_4.03/CTCAE_4.03_2010-06-14_ QuickReference_8.5×11.pdf .

16. National Cancer Institute NIH, US Department of Health and Human Services. (2017). Common terminology criteria for adverse events (CTCAE) version $5.0 \mathrm{https}$ ://ctep.cancer.gov/protocolDevelopment/electronic applications/docs/CTCAE_v5_Quick_Reference_8.5x11.pdf .

17. Lacouture, M. E., Maitland, M. L., Segaert, S., et al. (2010). A proposed EGFR inhibitor dermatologic adverse event-specific grading scale from the MASCC skin toxicity study group. Support Care Cancer, 18(4), 509-522.

18. Lisi, P., Bellini, V., \& Bianchi, L. (2014). The epidermal growth factor receptor inhibitor-related skin toxicity index (EGFRISTI): A new tool for grading and managing skin adverse reactions to epidermal growth factor receptor inhibitors. Oncology., 87(5), 311-319.

19. De Tursi, M., Zilli, M., Carella, C., et al. (2017). Skin toxicity evaluation in patients treated with cetuximab for metastatic colorectal cancer: A new tool for more accurate comprehension of quality of life impacts. Oncology Targets of Theraphy, 10, 3007-3015.

20. Basch, E., Reeve, B. B., Mitchell, S. A., et al. (2014). Development of the National Cancer Institute's patient-reported outcomes version of the common terminology criteria for adverse events (PRO-CTCAE). Journal of the National Cancer Institute, 106(9), dju244.

21. Basch, E., Deal, A. M., Kris, M. G., et al. (2016). Symptom monitoring with patient-reported outcomes during routine cancer treatment: A randomized controlled trial. Journal of Clinical Oncology, 34(6), 557-565.

22. Dueck, A. C., Mendoza, T. R., Mitchell, S. A., et al. (2015). Validity and reliability of the US National Cancer Institute's patient-reported outcomes version of the common terminology criteria for adverse events (PROCTCAE). JAMA Oncology, 1(8), 1051-1059.

23. Bennett, A. V., Dueck, A. C., Mitchell, S. A., et al. (2016). Mode equivalence and acceptability of tablet computer-, interactive voice response system-, and paper-based administration of the U.S. National Cancer Institute's patient-reported outcomes version of the common terminology criteria for adverse events (PRO-CTCAE). Health and Quality of Life Outcomes, 14, 24.

24. Arnold, B., Mitchell, S. A., Lent, L., et al. (2016). Linguistic validation of the Spanish version of the National Cancer Institute's patient-reported outcomes version of the common terminology criteria for adverse events (PRO-CTCAE). Support Care Cancer, 24(7), 2843-2851.

25. Wagner, L. I., Berg, S. R., Gandhi, M., et al. (2013). The development of a functional assessment of cancer therapy (FACT) questionnaire to assess dermatologic symptoms associated with epidermal growth factor receptor inhibitors (FACT-EGFRI-18). Support Care Cancer, 21(4), 1033-1041.

26. Zubrod, C. G. S. M., Frei, E., Brindley, C., Gold, G. L., Shnider, B., Oviedo, R., Gorman, J., Jones, R., Jonsson, U., Colsky, J., Chalmers, T., Ferguson, B., Dederick, M., Holland, J., Selawry, O., Regelson, W., Lasagna, L., \& Owens, A. H. (1960). Appraisal of methods for the study of chemotherapy of cancer in man: Comparative therapeutic trial of nitrogen mustard and triethylene thiophosphoramide. Journal of Chronic Diseases, 11(1), 7-33.

27. Cella, D. F., Tulsky, D. S., Gray, G., et al. (1993). The functional assessment of cancer therapy scale: Development and validation of the general measure. Journal of Clinical Oncology, 11(3), 570-579.

28. Hays, R. D., Farivar, S. S., \& Liu, H. (2005). Approaches and recommendations for estimating minimally important differences for health-related quality of life measures. COPD., 2(1), 63-67.

29. Oken, M. M., Creech, R. H., Tormey, D. C., et al. (1982). Toxicity and response criteria of the eastern cooperative oncology group. American Journal of Clinical Oncology, 5(6), 649-655.

30. Cohen, J. (1960). A coefficient of agreement for nominal scales. Educational and Psychological Measurement, 20, 37-46.

31. Cohen, J. (1988). Statistical power analysis for the behavioral sciences. Hillsdale: Lawrence Erlbaum Associates. 
32. Norman, G. R., Sloan, J. A., \& Wyrwich, K. W. (2003). Interpretation of changes in health-related quality of life: The remarkable universality of half a standard deviation. Medical Care, 41(5), 582-592.

33. Reeve, B. B., Wyrwich, K. W., Wu, A. W., et al. (2013). ISOQOL recommends minimum standards for patient-reported outcome measures used in patient-centered outcomes and comparative effectiveness research. Quality of Life Research, 22(8), 1889-1905.

34. Cronbach, L. (1951). Coefficient alpha and the internal structure of tests. Psychometrika., 16(3), 297-334.

35. Nunnally, J. (1978). Psychometric theory (2nd ed.). New York: McGaw-Hill Book Company.

36. Cohen, J. (1968). Weighted kappa: Nominal scale agreement with provision for scaled disagreement or partial credit. Psychological Bulletin, 70(4), 213-220.

37. Chren, M. M. (2012). The Skindex instruments to measure the effects of skin disease on quality of life. Dermatologic Clinics, 30(2), 231-236 xiii.

38. Finlay, A. Y., \& Khan, G. K. (1994). Dermatology life quality index (DLQI)--a simple practical measure for routine clinical use. Clinical and Experimental Dermatology, 19(3), 210-216.

\section{Publisher's Note}

Springer Nature remains neutral with regard to jurisdictional claims in published maps and institutional affiliations.

\section{Submit your manuscript to a SpringerOpen ${ }^{\circ}$ journal and benefit from:}

- Convenient online submission

- Rigorous peer review

- Open access: articles freely available online

High visibility within the field

- Retaining the copyright to your article

Submit your next manuscript at $\boldsymbol{\nabla}$ springeropen.com 